Ce texte est la version auteur de I'article suivant: I. Guérin, I. Hillenkamp, C. Verschuur, "L'économie solidaire sous le prisme du genre : une analyse critique et possibiliste ", Revue française de socioéconomie, 22, 2019, p. 105-122.

\title{
L'économie solidaire sous le prisme du genre : une analyse critique et possibiliste
}

Isabelle Guérin, IRD-Cessma

Université Paris Diderot - CESSMA

Boîte Postale 7017 - 75205 Paris cedex 13

Isabelle Hillenkamp, IRD-Cessma

Université Paris Diderot - CESSMA

Boîte Postale 7017 - 75205 Paris cedex 13

Christine Verschuur, IHEID

Maison de la Paix, Chemin Eugène-Rigot 2, 1202 Geneva, Suisse

\section{Résumé}

Cet article propose d'étudier l'économie solidaire sous le prisme du genre à partir d'un double regard, critique et possibiliste. Ce double regard, inspiré à la fois de l'économie substantive polanyienne et des recherches féministes, éclaire la manière dont les pratiques d'économie solidaire renouvellent l'économique et le politique, compris ici à la fois comme catégories d'action et de pensée. II met aussi en lumière les chemins multiples et sinueux de l'émancipation, fruit d'un entremêlement entre principes d'échange dont l'équilibre est en renouvellement permanent.

Mots clefs : économie solidaire, genre, pouvoir, Polanyi, reproduction

The Gender of Solidarity economy: a critical and possibilist view

This paper offers a gender analysis of solidarity economy which combines a critical and a possibilist view. This double perspective is inspired both by the substantive meaning of economics suggested by Polanyi and by feminist scholarship. It sheds light on how solidarity economy practices renew economics and politics, understood as categories of action and thought. This double perspective also highlights the multiple and complicated paths of emancipation, which result from the intertwining between principles of exchange which balance is in constant flux.

Key words: solidarity economy, gender, feminism, power, Polanyi, reproduction,

L'économie solidaire est souvent une économie animée par les femmes ou pour les femmes. En France par exemple, $66 \%$ des salarié.e.s de l'économie sociale et solidaire (entendue ici au sens légal, associations, coopératives, mutuelles et fondations) sont des femmes (Observatoire national de I'ESS-CNCRES, 2012). Au Brésil, près de $44 \%$ des membres des quelques 20000 
initiatives retenues dans le Système d'information en économie solidaire lors du recensement effectué en 2010-2012 sont des femmes, mais cette proportion est certainement sous-estimée (Nobre, 2015). On observe aussi que les secteurs d'activité sont fortement genrés. Les services collectifs de restauration sont en large partie voire exclusivement féminins (Anderson, 2015 ; Ndoye, 2014), tout comme le sont les coopératives de transformation de produits alimentaires dans les quartiers populaires (Ypeij 2002, Hainard et Verschuur 2005). Les femmes sont aussi très actives dans certaines formes de commerce équitable (Charlier 2006, Saussey, 2012), les coopératives de consommation ${ }^{1}$, les clubs de troc et les monnaies sociales (Saiag, 2015). Elles sont présentes en majorité dans les activités d'amélioration de l'environnement et de l'habitat dans les quartiers - espaces verts, construction populaire, assainissement, gestion des déchets, gestion de l'eau (Bisilliat 1995, de Suremain 2005, Haritas 2015, Verschuur 2005, 2012), les associations ou coopératives d'appui à la petite enfance ou aux personnes âgées (de Suremain 1996, Fournier 2013, ILO 2015) ou encore les mutuelles de santé et d'épargne-crédit (Chatterjee 2014, Fonteneau 2015). En milieu rural, les femmes sont très impliquées dans les collectifs de production agricole de subsistance (León 1980, Angulo 2011, Morales 2012 ; Guétat-Bernard et Saussey 2014) et en particulier d'agro-écologie (Hillenkamp, Nobre et al. 2016. Prévost 2015).

Ce constat est peu surprenant. II ne fait que refléter la dimension genrée du travail, que l'on trouve aussi bien dans les espaces privés que publics, domestiques, "communautaires " ou marchands (Lévesque 2005). Nombre d'initiatives d'économie solidaire portent sur les activités liées à la reproduction élargie de la vie, comme la préparation de l'alimentation, le nettoyage, les soins aux autres ou à la nature. Or ces activités, on le sait, restent l'apanage des femmes.

La question centrale, encore peu posée, est celle des implications de cette surreprésentation féminine. Est-ce une illustration supplémentaire de la surcharge de travail et de la sousvalorisation du rôle des femmes et un mécanisme de sa reproduction ? Cette question est d'autant plus légitime que l'emploi associatif, qui caractérise une large partie de l'économie solidaire, est souvent de faible qualité en termes de niveau de salaire et de conditions d'emploi ${ }^{2}$. Ou bien faut-il voir dans cette surreprésentation féminine l'amorce de formes novatrices de création de richesses, plus solidaires et plus égalitaires? En d'autres termes, l'économie solidaire est-elle seulement féminine ou peut-elle aussi être féministe, et surtout, à quelles conditions?

Dans le prolongement de réflexions antérieures ${ }^{3}$, complétées et nourries de travaux empiriques récents présentés et discutés à l'occasion d'un colloque organisé sur le sujet ${ }^{4}$, cet article propose une contribution à ce débat. Nous suggérons tout d'abord qu'un double regard, critique et possibiliste, est nécessaire (\$1), attentif à la fois à la violence de la domination et à ses contestations, aux effets de structures et à leurs interstices. Ce double regard, inspiré de l'économie substantive polanyienne, ainsi que de certaines recherches féministes, montre comment les pratiques d'économie solidaire renouvellent à la fois l'économique (\$2) et le politique (§3), compris ici comme catégories d’action et de pensée. II met aussi en lumière la diversité des luttes, et surtout les chemins multiples et sinueux de l'émancipation (\$4). Ce double regard, enfin, suggère d'appréhender l'émancipation comme étant indissociable de l'articulation entre principes d'échange polanyiens (marché, redistribution, réciprocité et partage domestique), celle-ci étant comprise non pas comme une illusoire quête d'équilibre,

\footnotetext{
${ }^{1}$ Au Japon, 80\% des 27 millions de membres de coopératives de consommation sont des femmes (ILO nd).

2 Pour un exemple français, voir Dussuet et Flahault (2012).

${ }^{3}$ Références supprimées pour anonymat.

${ }^{4}$ Référence des actes du colloque supprimée pour anonymat.
} 
mais comme un processus sous tension et donc sans cesse renouvelé (§5). II nous semble que ce renouvellement permanent ne saurait être considéré comme une fragilité, mais comme une condition même de l'existence et de la pérennisation de ces initiatives.

\section{Un regard critique et possibiliste}

Le regard critique consiste à étudier la nature des rapports sociaux qui animent les pratiques d'économie solidaire et leur articulation avec les rapports sociaux dominants. II interroge la manière dont les initiatives libèrent les femmes d'espaces clos ou au contraire les y enferment, la manière dont elles renforcent ou suscitent des inégalités non seulement de genre, mais aussi de classe, de race et d'ethnicité. II questionne leur éventuelle substitution, à moindre frais, à l'intervention publique, qu'elle soit locale, nationale ou supranationale ou encore la manière dont elles se transforment en réceptacle de chaînes de valeur globalisées, voire de mouvements religieux extrémistes. Se focaliser sur les rapports de domination a toutefois plusieurs écueils. On court alors le risque d'ignorer " la capacité d'action culturelle des sujets » et de " déserter le terrain des luttes sociales réelles" (Verschuur et Destremau, 2012, p. 9). II entraîne également un "fondamentalisme de l'alternatif ", lequel conduit à "à rejeter des propositions qui, nées au sein du capitalisme, ouvrent toutefois la voie à une orientation non capitaliste et créent des enclaves de solidarité au sein du système " (Sousa Santos et Rodriguez 2013, p. 133 ; voir aussi Dacheux et Goujon, 2011, chap. 3).

C'est pourquoi l'analyse critique mérite d'être combinée à un regard utopique, explorant les virtualités en visant "le dépassement des limites sociales et économiques imposées dans la réalité " (Cattani 2006b, p. 653). Cette posture utopique, que l'on peut qualifier également de "possibiliste " consiste à substituer une " herméneutique de l'émergence " (Sousa Santos et Rodriguez 2013) à celle du scepticisme (ibid.), à adopter la "passion du possible " pour s'autoriser à percevoir la part de changement inattendu et même improbable au-delà des régularités sociales (Hirschman, 1971). En d'autres termes, ce double regard consiste à reconnaître, et à assumer, une tension irréductible entre l'horizon émancipateur et le caractère graduel, hésitant et parfois ambigu des changements.

Ce double regard ne va pas de soi puisqu'il suppose de combiner des traditions épistémologiques souvent pensées comme contradictoires, attentives d'une part aux subjectivités, aux mondes vécus, aux marges de manœuvre, aux résistances et aux processus de changement et d'autre part aux structures et aux rapports de pouvoir, source de différentiation et de domination. Ce double regard impose par ailleurs de s'affranchir de nos catégories d'analyse, trop souvent figées dans une conception abstraite et autonome, séparée, de l'économique, du social et du politique. Socioéconomie et féminisme sont ici particulièrement féconds.

Tout d'abord, l'approche substantive de l'économie inspirée des travaux de Karl Polanyi (1983) offre des outils précieux pour analyser les institutions, les rapports sociaux et les choix politiques qui orientent la manière dont chaque société organise les activités de production, d'échange, de financement et de consommation. En reconnaissant plusieurs principes d'échange et d'interdépendance - le marché, la redistribution, mais aussi la réciprocité et le householding (partage domestique) - cette approche porte un regard possibiliste sur les multiples manières de " faire de l'économie » en combinant ces différents principes. Cette approche autorise dans le même temps une analyse critique de l'institution de l'économie dans la société, en particulier 
depuis un point de vue féministe (Waller et Jennings, 1991 ; Benería, 1998) et dans une visée démocratique (Hillenkamp et Laville, 2013). L'approche de la diversité institutionnelle, dans la lignée d'Elinor Ostrom (2005), permet également d'envisager les communs comme des institutions hybrides, dépendant d'un ensemble de règles d'accès et d'usage des ressources forgés dans des rapports sociaux particuliers. Des configurations extrêmement différentes peuvent en résulter, allant d'une gouvernance démocratique et solidaire des communs, à un adossement à la gestion publique, à l'exclusion de groupes ou d'individus de l'usage de certaines ressources ou encore à la division de ces dernières pour un usage individuel (Servet, 2014). Des possibilités d'émancipation et de reproduction des inégalités et de l'exclusion se nichent dans l'entrelacement de ces diverses logiques et institutions forgées à différents niveaux.

Cette première grille mérite ensuite d'être combinée à une perspective féministe. Depuis leur origine, les études féministes n'ont eu de cesse de déconstruire les catégories dominantes en dénonçant leur dimension à la fois sexuée, hiérarchique et normative, ainsi que les angles morts et les inégalités qui en résultent. Au-delà de la singularité des différents courants (et des conflits qui en résultent), plusieurs éléments peuvent être retenus pour notre propos.

Ces études ont montré en quoi le travail domestique, gratuit et invisible est un travail comme les autres (Combes et Devreux 1985 ; Delphy 1970). Revisitant l'histoire, elles ont réhabilité le rôle des femmes, non seulement dans les processus d'industrialisation mais dans les luttes ouvrières (Tilly et Scott 1978). Elles ont contesté la séparation arbitraire et fallacieuse entre production et reproduction, et souligné à quel point le maintien de rapports sociaux de type domestique conditionne et nourrit l'accumulation de type capitaliste (Meillassoux 1975, 1984). Avec le féminisme post-colonial, elles ont déconstruit la catégorie "femme", montrant comment l'appartenance de sexe, et les inégalités qui en découlent, s'articule et se combine avec des inégalités de classe, de race, de religion, de préférence sexuelle, etc. Elles ont également déconstruit l'idée d'émancipation, en soulignant l'étroitesse mais aussi l'arbitraire de définitions normatives centrées sur l'autonomie et le salariat (Mohanty 1988). Elles ont également montré les apories de la "solidarité féminine » et dénoncé le "triple rôle " des femmes, sommées de combiner non seulement vie familiale et reproduction, vie professionnelle et production mais aussi vie communautaire et gestion de communs (Destremau 2013 ; Kabeer 1994). Elles ont également montré les ambigüités, et dans de nombreux cas les impasses, de politiques dites de développement visant à appuyer ces collectifs féminins. Au nom d'une prétendue émancipation, l'appui s'est souvent traduit par une instrumentalisation, une dépossession, et une nouvelle forme de captation d'une force de travail gratuite (Molyneux, 2007).

Dit autrement, les analyses féministes élargissent le spectre de l'oppression et de l'émancipation à l'ensemble des principes d'échange et permettent une relecture plus nuancée des principes polanyiens (Fraser 2010). Ce serait une erreur d'idéaliser l' " encastrement ", entendu comme situation subordonnant le marché à la réciprocité, et/ou à la redistribution et/ou au partage domestique, puisque ces principes se présentent aussi sous des modalités oppressives. Ce serait également une erreur de vilipender le désencastrement, qui subordonne la réciprocité, la redistribution et/ou le partage domestique au principe de marché, puisque ce dernier peut être porteur d'émancipation.

Ce double regard, critique et possibiliste, est exigeant au niveau conceptuel mais aussi empirique, puisqu'il suppose des méthodes d'observation diachroniques, attentives aux émergences et au renouvellement incessant des initiatives. Il est néanmoins indispensable pour s'affranchir des éternels débats sur l'économie solidaire, appréhendée tantôt comme un maigre 
palliatif aux failles de l'État ou du marché, tantôt comme moteur de transformation : l'enjeu consiste à la fois à s'ouvrir à une multiplicité d'initiatives et de chemins d'émancipation sans perdre de vue l'analyse critique du sens de leur action et des rapports sociaux sous-jacents.

\section{Repenser l'économique}

Certaines approches féministes revendiquent de longue date une nouvelle conception de l'économie, comme ensemble de pratiques mais aussi comme savoir. L'économie est habituellement définie comme " science " des choix ou mode d'allocation des ressources rares. Elle occulte ainsi tout un pan de pratiques, notamment celles qui relèvent de la reproduction et du lien social. Ce faisant, elle rend invisible l'essentiel des activités assumées par les femmes (Nelson, 1993), mais aussi et plus largement l'ensemble des transactions, des relations et des motivations qui soutiennent pourtant une large part de l'activité économique, qu'il s'agisse de coercition, de dépendance, d'exploitation mais aussi de réciprocité, de partage, de solidarité, de confiance, de coopération, d'engagement, de spiritualité, etc. (Gibson-Graham, 2014).

En s'impliquant, localement, dans ce qui est indispensable pour l'entretien et la reproduction des communautés et des territoires (Degavre, 2011), nombre d'initiatives de femmes revisitent la manière dont sont articulées la " production " et la " reproduction ». Elles contestent ainsi en pratique une séparation arbitraire du point de vue de leur expérience vécue, contestée par les féministes, et qui avait été déterminée en fonction des exigences du développement et de la prospérité de l'économie capitaliste dominante (Meillassoux 1975 ; Federici 2010).

Cette ré-articulation prend des formes diverses selon les contextes et les périodes de I'histoire. Dans différents pays d'Amérique du Sud et d'Afrique de l'Ouest, les cantines populaires mutualisent la préparation des repas, contribuant ainsi à la sécurité alimentaire des familles tout en soulageant les femmes d'une partie de leur travail domestique (Angulo, 2011 ; Ndoye, 2014). Dans de nombreux contextes, la préparation des repas est chronophage pour différentes raisons - pas ou peu d'équipement, traditions culinaires supposant des temps longs de préparation et de cuisson. A cela se rajoutent des facteurs chroniques ou conjoncturels d'insécurité alimentaire. Dans une perspective de gain de temps et de coût, des femmes se regroupent pour cuisiner collectivement et alternativement. Elles mettent parfois l'accent sur les circuits courts, dans une perspective de souveraineté alimentaire locale, comme par exemple au Sénégal (Ndoye, 2014). Lorsqu'elles sont spontanées, ces initiatives des femmes sont parfois relayées par des entités extérieures, dont des politiques publiques. Y compris dans ce cas, elles peuvent développer un potentiel émancipatoire lorsque la mise en place de l'activité s'accompagne de la construction d'un sujet collectif. En France, les restaurants de quartier permettent à des femmes subalternes, souvent d'origine immigrée, d'exercer une activité rémunérée compatible avec leurs responsabilités domestiques, tout en (re)créant des lieux de vie dans des quartiers désertés et disqualifiés (Hersent et Soumbou, 2011).

L'exemple péruvien met bien en évidence les possibilités et les limites de ces initiatives et l'intérêt d'une approche à la fois critique et possibiliste. A leur pic de fonctionnement à la fin des années 1990, on estimait que les cuisines péruviennes de Lima servaient environ 1,4 millions de repas quotidiens (Anderson, 2015). Ce mouvement, initié dans les années 1970 et encore d'actualité aujourd'hui, a fait l'objet de nombreuses controverses. Romantisé par certain.es au nom d'une prétendue émancipation individuelle et collective pour les femmes de milieux 
populaires, il a été accusé par d'autres de maternalisme et de conservatisme, les femmes pauvres étant finalement condamnées à se prendre en charge et à assumer par elles-mêmes des questions d'intérêt général. L'histoire, la trajectoire et les ambigüités multiples des cuisines collectives péruviennes ont largement été documentées (ibid.). On soulignera simplement ici que ces initiatives ont permis aux femmes de sortir de l'enfermement domestique et de publiciser la question de la sécurité alimentaire et de la préparation des repas. Si les soutiens n'ont jamais été à la hauteur de leurs attentes et revendications, s'ils se sont parfois traduits par des récupérations politiques et instrumentalisations diverses, les cuisines ont néanmoins bénéficié de subventions publiques, certes limitées ${ }^{5}$, mais reconnaissant leur contribution d'utilité générale. Par ailleurs, bien au-delà de la préparation des repas, les cuisines représentent des lieux de socialisation, d'échange et d'apprentissage, comme le montrent différentes monographies (Blondet, 1995; Angulo, 2011; Anderson, 2015). Certain.es argueront que les cuisines ne règlent en rien l'inégale division sexuelle du travail. C'est effectivement le cas, mais permettre aux femmes de sortir de l'enfermement du rapport social de type domestique est déjà une avancée (Lipietz, 2002).

La séparation entre "production " et "reproduction ", dénoncée de longue date par les recherches féministes, n'a plus lieu d'être. Ces deux pans de la réalité sont articulés au quotidien dans des pratiques qui visent la sécurité des moyens d'existence des femmes et de leurs familles et la revitalisation de communautés et d'identités locales. Une lecture féministe invite à reconnaître le rôle essentiel des activités dites " de reproduction ", qui sont tout aussi " productives " que les activités labellisées ainsi mais circonscrites aux activités créatrices de valeur matérielle, et sans lesquelles celles de "production " ne pourraient avoir lieu. Plus encore, une lecture féministe (et possibiliste) fait valoir leur rôle fondamental en matière de cohésion sociale et d'épanouissement individuel et collectif. Certaines initiatives d'économie solidaire ont précisément pour objet de rendre visible le travail féminin, de mettre en évidence son rôle essentiel en matière de création de richesse, éventuellement de le quantifier mais aussi de plaider pour de nouveaux rapports de production et de nouvelles conceptions de la richesse qui rendraient obsolètes le clivage production / reproduction.

Si l'on reprend l'exemple des cantines Péruviennes, et comme nous l'avons souligné plus haut, en dépit de toutes leurs faiblesses, ces initiatives ont eu le mérite de mettre sur la place publique les problèmes récurrents de sécurité alimentaire et le rôle décisif des femmes dans la gestion de ce problème au quotidien (Basu, 1995, p. 8). Au Sénégal, les initiatives de restauration de quartier, plus récentes, poursuivent un combat similaire (Ndoye, 2014). Dans un contexte de mépris à l'égard des activités de rue, les femmes se battent non pour bénéficier de financements publics mais simplement pour exister et éviter d'être "déguerpies". Calculer leur contribution monétaire à l'économie locale est une manière de revendiquer leur droit à l'existence.

Cet exercice de valorisation existe aussi pour des activités dégradantes et pourtant essentielles, comme le ramassage des déchets. Dans la ville de Pune en Inde, un syndicat de femmes ramasseuses de déchets a construit sa légitimité sur la valorisation de ce type de travail, et ceci tant à l'égard de ses propres membres, situées au plus bas de la hiérarchie sociale et elles-mêmes convaincues de la « saleté » de leur statut, que des autorités publiques. Calculer l'équivalent monétaire du travail effectué a mis en évidence les économies

\footnotetext{
${ }^{5}$ D'après Angulo (2011), le subventionnement public représente moins de $20 \%$ de leurs coûts alors qu'elles en revendiquent $60 \%$.
} 
considérables accomplies par la municipalité sur le traitement des déchets (330 000 US\$ par an, chaque travailleuse réalisant mensuellement l'équivalent de $5 \$$ de travail gratuit). $D$ 'autres calculs visent à démontrer la contribution de la collecte à l'économie locale, le financement indirect du processus de recyclage ou encore les gains d'énergie réalisés par rapport à des formes mécaniques de collecte (Narayan et Chikarmane, 2013).

Au-delà d'exercices de quantification en équivalent monétaire, c'est aussi la valorisation sociale et symbolique de la composante reproductive qui est mise en avant : celle-ci n'est plus considérée comme une contrainte mais comme une fin en soi, dissipant ainsi l'opposition factice entre production et reproduction. L'objectif n'est pas d'accumuler, mais de mener des activités "visant la reproduction, au niveau de la société, du lien social, de la subsistance matérielle et, plus globalement, d'un territoire de vie " (Degavre, 2011, p. 82). L'exemple du groupe de recherche-action Community Economies est ici illustratif. Partant du principe que tous les territoires disposent de ressources insoupçonnées, trop souvent occultées, disqualifiées ou méprisées par une pensée dominante, monoculturelle et "capitalocentrée ", le groupe prône une approche non pas en termes de manques et de déficiences (pauvreté, chômage, faible capital humain, faible productivité, etc.) mais de valorisation des ressources comprises au sens large : celles qui œuvrent au bien-être individuel et collectif, qui distribuent un surplus de nature matérielle mais aussi sociale et culturelle et qui construisent et entretiennent des communs (Gibson-Graham, 2005, p. 16 ; voir aussi Federici 2010). II s'agit de construire, de manière transversale, des rapports sociaux de type solidaires et démocratiques.

\section{Repenser le politique}

Tout comme l'économique, le politique a été victime de catégories normatives et genrées, fondées sur des conceptions singulières du public et du privé, occultant la complexité des pratiques quotidiennes et tout un répertoire de formes d'engagement, notamment, quoique non uniquement, celles des femmes. Dans ces schèmes de pensée dominants, débat public et action politique ne seraient possibles qu'en s'émancipant des intérêts dits "privés ", que ceux-ci relèvent de la sphère domestique ou de la sphère marchande (Waller et Jennings, 1991). Battant en brèche ces oppositions, l'histoire féministe a permis de réhabiliter des formes diverses d'engagement, en montrant que ce sont souvent les responsabilités domestiques des femmes qui les amènent à s'engager dans le combat politique ${ }^{6}$. Cette relecture du politique, compris non plus comme un champ autonome mais indissociable de l'action et de la sphère privée, et recouvrant dès lors une multiplicité de pratiques, permet de mettre en évidence le contenu éminemment politique des initiatives d'économie solidaire, y compris lorsqu'elles sont animées par des femmes.

Cette politisation s'exerce à des niveaux multiples, depuis la création d'espaces micro-locaux de délibération qui sont essentiels dans l'identification des priorités et des modes d'action jusqu'à des tentatives d'interpellation aux échelons nationaux et internationaux ${ }^{7}$. Loin d'être hiérarchisés, ces échelons interagissent entre eux, le global étant bien souvent conditionné par le local. Cette politisation prend des formes également multiples, oscillant entre mise en débat public négociation et dialogue, jusqu'à des formes plus radicales de contestation (Guérin et Nobre, 2015). Prenons l'exemple des mutuelles de santé, qui se sont fortement

\footnotetext{
${ }^{6}$ Voir par exemple Tilly (1978).

${ }^{7}$ Pour différents exemples, voir Guérin (2003), Guérin, Hersent et Fraisse (2011), Charlier (2006), Verschuur Guérin et Hillenkamp (2015).
} 
développées au cours des deux dernières décennies en Afrique de l'Ouest mais aussi dans plusieurs pays d'Amérique du Sud. Leurs limites et faiblesses ont largement été documentées (faible capacité de couverture, homogénéité des membres ne permettant guère une réelle répartition des risques, exclusion des plus démuni.e.s, etc.). Ce sont là de bien piètres substituts à des systèmes universels et réellement redistributifs de protection sociale, peuton également arguer. Outre le fait que ces mutuelles ont eu pour mérite initial de répondre ici et maintenant à des problèmes dont personne ne se souciait, elles ont joué un rôle évident dans la mise en débat public puis dans l'élaboration de systèmes de protection sociale qui se veulent universels, notamment en Afrique sub-saharienne (Fonteneau et al. 2014, pp. 22-27).

Au sein des mouvements comme celui des Piqueteros en Argentine (Rauber 2002), des émeutes de la faim, ou des Indignés, les femmes ont souvent joué un rôle de premier plan. Or ces mouvements ont pour particularité d'articuler révolte et expérimentation: la dénonciation de mécanismes d'oppression et d'exploitation s'accompagne d'actions concrètes qui offrent des avantages matériels aux participant.e.s tout en leur permettant de construire les répertoires d'action et les solidarités qui sont nécessaires aux moments de révolte. Ces luttes politiques ne sont possibles qu'articulées avec des pratiques permettant d'assurer la survie quotidienne de celles et ceux qui y sont engagés. Les activités de reproduction sociale - dans leur dimension matérielle mais aussi conviviale et festive (Nobre, 2015), - conditionnent ainsi l'engagement politique.

\section{Les chemins multiples et sinueux de l'émancipation}

La posture possibiliste exige de redéfinir les catégories, à commencer par celle de l'émancipation. Qui définit l'émancipation et au nom de qui ? S'agit-il de rompre radicalement avec l'ordre du divin et du religieux et d'affirmer le primat de l'individu, de la raison et du contrat ? De se libérer de toutes les structures d'oppression, qu'elles soient d'ordre matériel, social, symbolique ou encore sexuel ? De se départir d'un inconscient refoulé, de ses propres inhibitions et traumatismes psychiques? Ou encore d'être reconnu.e, sous la forme de liens affectifs, de droits légaux ou de statut social ? L'émancipation est-elle d'abord individuelle ou collective, humaine ou politique ? Dès lors que l'on considère l'économie solidaire depuis la perspective de la reproduction, l'analyse des alternatives et du changement social dans l'économie plurielle s'enrichit de nouveaux questionnements. La question n'est plus seulement celle de la viabilité et du potentiel émancipateur de formes d'organisation de la production, du financement, des échanges ou de la consommation fondées sur l'égalité, la solidarité ou la protection de l'environnement (Sousa Santos et Rodríguez 2013). Elle est, plus largement, celle de chemins multiples et corrélés d'émancipation. Ceux-ci peuvent emprunter des voies "classiques ", associées à la réorganisation de la sphère de la production et à ses processus d'institutionnalisation et d'autres, plus novateurs, surgissant de la démocratisation de la sphère de la reproduction et de la contestation de la séparation et de la hiérarchisation entre production et reproduction.

Cette multiplicité des chemins d'émancipation est également liée à la complexité des identités et des sources d'oppression, y compris au sein de contextes singuliers. Dès lors que l'oppression est multiple, alors les chemins d'émancipation et les luttes qui y conduisent le sont également. Cette approche fait écho au modèle de démocratie "plurielle " et " radicale " (Laclau et Mouffe, 2009). Partant du constat de la multiplicité des rapports de domination, ce modèle postule que l'engagement dans la sphère publique est une condition essentielle des libertés individuelles et de la définition de celles-ci. 
Seules la contextualisation et l'historicisation permettent de saisir pleinement le sens et la portée de ces luttes multiples. La définition et la forme des actions à mener varient en fonction des priorités, des identités et des enjeux locaux. En Amérique latine, les revendications féministes dans les mouvements d'economía solidaria se sont d'abord inscrites dans des enjeux beaucoup plus larges, en particulier ceux liés à la question de l'intégration régionale, le lobbying contre la zone de libre-échange des Amériques (ZLEA, ou ALCA : Área de libre comercio de las Américas) et la dénonciation de l'impérialisme américain (Nobre \& Freitas 2011). Au Pérou, nous l'avons vu plus haut, les cantines populaires autogérées luttent contre l'invisibilité du travail féminin mais aussi contre l'insécurité alimentaire que vit le pays depuis plusieurs décennies (Angulo, 2011). En Bolivie, les associations ou coopératives de productrices font partie d'un mouvement plus large de revendication de l'identité indigène qui s'est opposé, au début des années 2000, au régime néolibéral (Hillenkamp, 2013). En France, les initiatives de femmes des banlieues ne se battent pas contre la domination masculine. Elles revendiquent le droit de produire et d'entreprendre autrement et elles méritent d'être resituées par rapport à la triple discrimination dont elles font l'objet : elles sont à la fois femmes, immigrées et pauvres (Hersent et Soumbou, 2011). D'autres initiatives, mêlant hommes et femmes, ont pour motivation première de "sécuriser " l'existence des femmes et de "résister ", par exemple en donnant du sens au travail ou en réparant le vivant (Saussey et Degavre, 2015). Dans la même veine, des luttes urbaines observées dans plusieurs métropoles visent d'abord et avant tout à améliorer le quotidien des habitants et à faire valoir l'accès réel aux droits de base, qui sont ignorés et bafoués (Verschuur, 2005 ; 2011). Dans diverses régions du monde, les collectifs de femmes travailleuses se mobilisent avant tout pour être reconnues, beaucoup plus que pour dénoncer l'emprise du capital (Kabeer et al. 2013). Cette lutte pour la reconnaissance s'enracine dans la construction d'une identité commune, mais celle-ci peu rarement être donnée a priori compte tenu de la fragmentation des travailleuses, notamment en termes de classe, de caste ou de race.

\section{L'entremêlement des principes d'échanges : une expérimentation permanente}

L'approche possibiliste révèle enfin la pluralité des principes d'échange et d'interdépendance entre les êtres humains: les pratiques marchandes concrètes sont systématiquement entremêlées à d'autres principes, qu'il s'agisse de réciprocité, de redistribution ou de partage domestique (Laville, 2013). Cette observation s'applique aussi aux communs, dès lors que l'on reconnait leur statut d'institutions hybrides, traversées par différents principes (Hillenkamp et Servet, 2015). Le " marché » et "l'Etat » sont souvent fétichisés, appréhendés de manière abstraite à partir de principes normatifs qui surestiment considérablement leur portée (considérée tantôt comme aliénante ou émancipatrice en fonction des écoles de pensée). Or l'un comme l'autre, en réalité, n'opèrent jamais de manière isolée. C'est précisément la nature de l'entremêlement entre principes d'échange et d'interdépendance qui définit la dimension plus ou moins émancipatrice ou oppressive des pratiques économiques. Cette économie plurielle est à la fois une réalité et un horizon possible de justice sociale. La communauté fait lien, donne du sens et protège, mais elle est traversée de rapports de pouvoir, lieu où s'expriment et se produisent exclusion et domination. La puissance publique redistribue, sécurise et reconnaît (parfois) les droits des individus, mais elle en nie les désirs. Le marché reconnaît et soutient les libertés, mais seulement ceux des mieux lotis, les autres sont exclus ou condamnés à être exploités et paupérisés. Les personnes, quelles qu'elles soient, où qu' elles soient, ont besoin de tout ceci à la fois. 
Depuis cette perspective, il apparait que le potentiel émancipateur des initiatives d'économie solidaire repose sur des configurations articulant les quatre principes de réciprocité, redistribution et partage domestique et marché en les soumettant à des principes de démocratie et d'égalité. Or, ces configurations plurielles sont instables, tout d'abord parce qu'elles s'ancrent bien souvent dans des asymétries structurelles de position qui reflètent et cristallisent des appartenances de classe, de race, de caste, de genre, etc. Du fait de l'adaptation permanente à des contextes à la fois singuliers et changeants, les modes et les mobiles de lutte sont une succession d'avancées et de reculs dont l'issue est souvent imprévisible (Kabeer et al., 2013). Lorsque les initiatives parviennent à s'inscrire dans la durée, leur survie et le maintien de leurs objectifs initiaux sont un combat quotidien. L'histoire des cantines péruviennes (Anderson, 2015), des Self-Help-Groups indiens (Sudarshan, 2015) et kenyans (Johnson, 2015) révèle d'étonnantes similitudes, alternant collaboration, confrontation et cooptation avec et par différentes entités ou réseaux institués, qu'il s'agisse des pouvoirs publics, des partis politiques et parfois d'entités religieuses. Cet équilibre est donc éminemment fragile et constamment soumis à l'épreuve, mais il est possible.

Face aux risques incessants de subversion et d'accaparement, certain.e.s activistes et intellectuel.le.s féministes revendiquent l'autonomie, à la fois institutionnelle et financière (Falquet, 2008). D'autres plaident pour l'existence d'espaces autonomes, seuls à même de permettre aux femmes de définir leurs propres priorités et stratégies, à l'abri d'intrusions extérieures (Molyneux, 2007, p. 394), mais qui ensuite peuvent être reliés à des luttes plus vastes. Cette volonté d'autonomie peut passer par un usage tactique des partenariats publics, comme le montre l'exemple de la Self Women Employment Association (SEWA) en Inde. Convaincue que l'Etat est incapable d'appuyer des initiatives sans les détourner de leurs objectifs initiaux, la SEWA refuse toute réplication à grande échelle des projets qu'elle initie (Chatterjee, 2014). L'association n'accepte la collaboration avec les pouvoirs publics qu'à l'échelon local ou territorial afin d'éviter la dissolution des spécificités locales. Ceci ne l'empêche pas, par ailleurs, de peser sur l'intervention publique par le biais de la législation et de la réglementation et ceci avec un certain succès, depuis la reconnaissance du travail féminin dans les années 1970 jusqu'à la question actuelle de la protection sociale.

Lorsque les luttes sont fragmentées ou que les populations locales sont hétérogènes, le recours à des entités extérieures est un moyen de les articuler, au moins partiellement, de les mettre en réseau et de dépasser certains clivages (Kabeer et al., 2013), autrement dit de les relier sans nécessairement les intégrer, dans le respect de la pluralité démocratique. En ce sens, le recours à des tiers (notamment ONG, universités, incubateurs, etc.) est inhérent au modèle de démocratie radicale et plurielle. Cependant, le rôle de ces tiers fait débat : doiventils être cantonnés à un rôle " médiateur " (Guérin, 2003) ou de "facilitateur " (Kabeer et al., 2013), ou bien doivent-ils être considérés comme des acteurs de plein pied, comme on peut l'observer dans plusieurs pays d'Amérique Latine, notamment le Brésil ? Dans ce cas, comment assurer une participation égalitaire d'acteurs très différents à des espaces publics eux-mêmes hétérogènes ? Etant donné les asymétries de pouvoir intrinsèques aux relations entre organismes " d'appui » et organisations " locales », expliciter ces asymétries, et non les occulter, est un premier rempart contre les risques de dérives (Solomon et al., 2013).

Cette recherche d'équilibre est un processus permanent, jamais abouti. S'il peut donner un sentiment d'inachèvement et "d'expérimentation permanente" (Hersent, 2014), il conditionne l'existence même de ces initiatives. Et il se traduit par une diversité 
d'arrangements possibles, qui prennent forme en fonction de contextes et de trajectoires locales et singulières.

\section{Conclusion}

La place donnée ici aux initiatives d'économie solidaire dans l'émancipation féminine mérite, pour conclure, d'être replacée dans une histoire plus longue des modèles et des acteurs présumés du changement social. Au cours du XIXe siècle, principalement durant sa seconde moitié, les mouvements sociaux défendant la lutte des classes se sont installés comme le moteur présumé de l'histoire. Au cours du XXe siècle, cette position a été questionnée par les études féministes qui ont fait remarquer combien l'analyse de la division sexuelle du travail et de la place des femmes en était absente : invisibles, les ouvrières; invisibles, les femmes des ouvriers organisés en collectifs et les femmes salariées ; invisibles, le travail de reproduction, qui permettait pourtant de maintenir les salaires des ouvriers à leur bas niveau. Il est devenu patent que l'absence de réflexion sur les rapports sociaux de sexe et le modèle familial qui soutenaient l'action des mouvements ouvriers limitait leur portée et entretenait le système patriarcal. Par la suite, les "nouveaux mouvement sociaux " ont mis en scène des actrices et acteurs inédits, renouvelant à la fois les formes d'action et les objets de conflits, autour de la défense des minorités et des droits. Ils ont interpellé, de l'extérieur et de l'intérieur, l'ancien mouvement ouvrier (Maruani 1979), mis en cause la distinction entre les mouvements sociaux d'orientation " redistributive " et classiste, et les mouvements "culturels " ou identitaires (féministes, anti-racistes...). Ils ont posé la question de l'opposition entre les politiques de reconnaissance et de redistribution, et de la réconciliation, ou non, de la gauche culturelle et de la gauche sociale (Fraser, 2005).

Dans le contexte actuel de globalisation et de financiarisation, nombre de repères habituels sont ébranlés. Les pratiques antérieures de mobilisation sont dépassées. En même temps, les organisations de la société civile, les "sans-pouvoir ", occupent de nouveaux espaces. Les changements sont réclamés " ici et maintenant ", sans attendre "le grand soir ». Les chemins de l'émancipation et des transformations sociales sont à ré-imaginer. Précisément, les initiatives décrites ici nourrissent cette nouvelle imagination dont nous avons tant besoin. Evidemment, ces initiatives ne font pas table rase des institutions qui ont forgé le champ de la protection et de la reproduction sociale depuis le XIXe siècle - en particulier les mutuelles et les assurances sociales - ni des formes d'exploitation qui se renouvellent, aujourd'hui notamment à travers la nouvelle division internationale du travail. Les femmes, et notamment les femmes de catégories subalternes, se voient déléguer des responsabilités croissantes d'activités de soin, tant à autrui qu'à l'égard de la nature.

Apprécier à sa juste valeur les capacités subversives et émancipatrices de l'économie solidaire suppose donc une grille de lecture adéquate. Combiner regard critique et possibiliste permet de mettre en lumière ce potentiel jusque-là insoupçonné, sans perdre de vue les rapports sociaux. Loin de se cantonner à des expériences fugaces et éphémères, les pratiques d'économie solidaire contribuent à repenser la notion même d'économie et à la transformer en pratique. Celle-ci ne se limite plus à la production ou l'allocation de ressources, mais recouvre l'ensemble des relations et activités nécessaires à la reproduction et l'entretien de la vie. Aux actions concrètes, les initiatives articulent des pratiques de délibération, de 
discussion, mais aussi parfois de mobilisation, de résistance, voire de révolte, contribuant ainsi à repenser le politique. Elles répondent en cela à des préoccupations anciennes de certaines mouvances féministes, convaincues que la lutte contre le patriarcat suppose de reconsidérer la nature même de l'économique et du politique, ainsi que l'hétérogénéité fondamentale des aspirations et des contraintes des femmes.

Est-il dès lors possible de se prononcer sur les conditions d'une économie solidaire féministe, et pas seulement féminine ? Que les femmes, et notamment les plus exclues, ainsi que les hommes, se (ré)approprient leur destinée et décident par eux-mêmes de leurs priorités et de leurs modes d'action est une condition incontournable, ce qui suppose d'admettre l'indissociabilité de l'action et de la délibération. Imaginer une discontinuité radicale entre, d'un côté, des alternatives émancipatrices et, de l'autre, des pratiques oppressives et sources d'exploitation est également illusoire. Nombre d'entre elles expérimentent de nouvelles manières de penser et de faire, tout en alimentant des dynamiques plus globales sur lesquelles elles n'ont pas toujours prise. Comme nous l'avons suggéré ici, identifier la nature de l'entremêlement entre principes d'interdépendance, entremêlement instable et en renouvellement perpétuel, est une manière de saisir la dimension plus ou moins émancipatrice ou oppressive de ces initiatives.

\section{Liste des références}

Agarwal, B. (2002). Le débat sur le genre et l'environnement. In D. Haase-Dubosc et al., (éd.), Enjeux contemporains du féminisme indien, (p. 155-180). Paris : MSH Editions.

Anderson J. (2015). Missed opportunities, mixed messages and lessons learned: collective kitchens in marginal urban communities in Peru. In Ch. Verschuur et al. (eds) Une économie solidaire peut-elle être féministe? Homo oeconomicus, mulier solidaria, (p. 221-242). Paris : L'Harmattan.

Angulo, N. (2011). Cantines populaires : sécurité alimentaire et exercice de la citoyenneté au Pérou. In I. Guérin, M. Hersent \& L. Fraisse (ed.), Femmes, économie et développement. De la résistance à la justice sociale, (p. 221-237). Paris : ERES/IRD.

Angulo, N. e.a (2011). Economía Social y Solidaria. Polítivas Públicas y Género. Buenos Aires, Asociación Lola Mora. AECID: 96

Basu, A. (éd.), (1995). The Challenge of Local Feminisms. Women's Movements in Global Perspective, New Delhi : Kali for Women.

Batliwala, S. \& Dhanraj, D. (2007). Gender Myths that instrumentalise Women : a View from the Indian Front Line. In A. Cornwall et al., (éd.), Feminisms in Development. Contradictions, Contestations and Challenges, (p. 21-34). Londres et New-York : Zed Books.

Benería L. (1998), « Karl Polanyi, la Construcción del Mercado Global y la "Diferencia" de Género ", Mientras Tanto, vol. 71, pp. 81-101.

Bisilliat, J. (1995). La construction populaire au Brésil: une expérience à São Paulo. Paris, Karthala.

Blondet, C. (1995). Out of the Kitchens and on to the Streets: Women's Activism in Peru ", In A. Basu (éd.), The Challenges of Local Feminisms. Women's Movements in Global Perspective, (p. 251-275). Boulder: Westview Press. 
Calvès, A.-E. (2009). "Empowerment": généalogie d'un concept clé du discours contemporain sur le développement." Revue Tiers Monde 200(4): 735-749.

Charlier, S. (2006). L'économie solidaire au féminin: quel apport spécifique pour l'empoderamiento des femmes? Une étude de cas dans les Andes boliviennes (Thèse de doctorat). Louvain la-Neuve : Presses universitaires de Louvain.

Charlier, S. (2011). Empoderamiento des femmes par l'économie populaire et solidaire: participation et visibilité des femmes en Bolivie. In I. Guérin, M. Hersent \& L. Fraisse (ed.), Femmes, économie et développement, Entre résistance et justice sociale, (p. 155-184). Paris : Erès/IRD.

Chatterjee M. (2014). Organising social protection through solidarity of women workers: experiences of the Self-Employed Women's Association, SEWA in India. In Ch. Verschuur, I., Guérin \& I. Hillenkamp I. (eds) (2015). Une économie solidaire peut-elle être féministe ? Homo oeconomicus, mulier solidaria, (p. 243-256). Paris : L'Harmattan.

Combes, D. and A.-M. Devreux (1992). Travail des femmes et rapports sociaux de sexe. in J. Bisilliat, F. Pinton and M. Lecarme (eds) Relations de genre et développement : femmes et sociétés. Paris, ORSTOM: 149-166.

Davis A. (1982). Women race and class. London. The Women's Press Ltd. traduction française 1983. Femmes. race et classe. Paris. Des Femmes

Degavre F. (2011). La pensée "femmes et développement ». In I. Guérin, M. Hersent \& L. Fraisse (ed.), Femmes, économie et développement, Entre résistance et justice sociale, (p. 6386). Paris : Erès/IRD.

Delphy Ch. (1970) L'ennemiprincipal. In Partisans, numéro spécial Libération des Femmes année 0 , novembre.

Destremau B., (2013), Au four, au moulin et à « l'empowerment ». La triple captation et exploitation du travail des femmes dans le développement, in : M. Maruani, (dir.), Travail et genre dans le monde, l'état des savoirs, Paris : La Découverte, p. 89-97.

Dussuet, A. \& Flahault, E. (2012). Des emplois soutenables dans les associations? La part du genre. Travail, organisations et politiques publiques: quelle "soutenabilité" à l'heure de la mondialisation ? Cahiers du Cirtes, Hors-Série n², 159-172.

Falquet, J. (2008). De gré ou de force. Les femmes dans la mondialisation. Paris : Éditions La Dispute, coll. "Le genre du monde ».

Federici, S. (2010). Feminism And the Politics of the Commons. In C. Hughes, S. Peace, \& K. Van Meter (éds.), Uses of a WorldWind, Movement, Movements, and Contemporary Radical Currents in the United States. Oakland: AK Press.

Fonteneau, B. Vaes S., Huyse H. (2014). Social Protection at the Top of the International Agenda: Issues at Stake from a Civil Society Perspective. HIVA-KU Leuven (Leuven)

Fonteneau, B. (2015). Extending social protection in health through SSE: possibilities and challenges in West Africa. In P. Utting (ed) Social and Solidarity Economy. Beyond the Fringe, (p. 250-265), London: Zed Books, UNRISD.

Fournier, M. L., et al. (2013). Género y economía social y solidaria. Hacia otra economía. V. Constanzo and D. Maidana. Buenos Aires, UNGS. 9. 
França Filho G. et Cunha E. (2009). "Incubação de redes locais de economia solidária: lições e aprendizados a partir da experiência do projeto Eco-Luzia e da metodologia da ITES/UFBA", Revista O\&S. Organizações \& Sociedade, vol. 16, pp. 725-47.

Gibson-Graham, J. K. (2003). Enabling ethical economies: Co-operativism and class. Critical Sociology. 29(2), 124-161.

Gibson-Graham, J. K. (2005). Surplus Possibilities: Postdevelopment and Community Economies. Singapore Journal of Tropical Geography, 26, 4-26.

Gibson-Graham, J. K. (2014). Rethinking the Economy with Thick Description and Weak Theory. Current Anthropology, 55(S9), S147-S153.

Guérin I., Hillenkamp, I. \& Verschuur, Ch. (2015). Homo oeconomicus, mulier solidaria. L'économie solidaire peut-elle être féministe ?, RECMA, 335, 7-14.

Guérin, I. (2003). Femmes et économie solidaire. Paris : La Découverte.

Guérin, I. (2015). La microfinance et ses dérives. Emanciper, exploiter ou discipliner ? Paris : Demopolis/IRD.

Guérin, I. \& Nobre, M. (2015). L'économie solidaire revisitée à la lumière du genre : outil de changement social ou reproduction de la subordination féminine ? In Ch.Verschuur, I. Guérin \& H. Guetat-Bernard, (dir.), Sous le développement, le genre. (p. 425-44). Marseille : Institut de recherche pour le développement (IRD).

Guérin, I., Hersent M. \& Fraisse L. (2011). Femmes, économie et développement, Entre résistance et justice sociale, Paris : Erès/IRD.

Guétat-Bernard H. Saussey M. (eds) Genre et savoirs. Pratiques et innovations rurales au Sud, Marseille : Editions de l'IRD.

Hainard, F. , Ch. Verschuur (2005). Mouvements de quartier et environnements urbains. La prise de pouvoir des femmes dans les pays du Sud et de l'Est. Paris, Karthala - ENDA.

Haritas K. (2015) In Verschuur et al. (eds). Une économie solidaire peut-elle être féministe? Homo oeconomicus, mulier solidaria. (p. 95-123). Paris : L'Harmattan

Hersent, M. (2014). Initiatives de femmes en économie solidaire et politiques publiques, Colloque 'Entreprendre au Pluri'elles : Femmes actrices de l'économie sociale et solidaire', Mouvement de l'économie solidaire, le Labo de l'ESS, l'Agence pour le développement de l'économie locale et le Centre interdisciplinaire de Recherche Travail, Etat et Société, Paris, Février 2014.

Hersent, M. \& Soumbou, P. (2011). Initiatives de femmes en migration dans l'économie solidaire. In I. Guérin, M. Hersent \& L. Fraisse (eds) Femmes, économie et développement. De la résistance à la justice sociale. (p. 207-220). Toulouse/Marseille : ERES/IRD.

Hillenkamp I. et Servet J.-M. (2015). (eds) Le marché autrement. Marchés réels et marché fantasmé, Paris, Classiques Garnier, coll. "Bibliothèque de l'économiste ».

Hillenkamp I., Laville J.-L., (2013), (éd.) Socioéconomie et démocratie. L'actualité de Karl Polanyi, Paris : Eres.

Hillenkamp, I. (2013). L'économie solidaire en Bolivie. Entre marché et démocratie. Paris, Genève: Karthala, GIP.

Hillenkamp, I. (2015). Solidarity Economy for development and women's emancipation. Lessons from Bolivia, Development and Change, 46(5), 1133-1158. 
Hillenkamp, I., Guérin, I. \& Verschuur, Ch. (2014). Economie solidaire et théories féministes: pistes pour une convergence nécessaire, Revue d'Economie solidaire, 7, Octobre, 5-43.

Hillenkamp, I., Nobre, M., avec la collaboration de Vivian Ferreira Franco, Daniela lanovalli, Gláucia Marques et Sheyla Saori (2016). Por uma economia solidária e feminista: pesquisaação no Vale do Ribeira. Papier présenté au Ve Symposium international "Desigualdades, direitos e políticas públicas: protagonismos e alternativas ", Universidade do Vale do Rio dos Sinos, São Leopoldo, Brésil.

Hirschman, A. (1971). A Bias for Hope: Essays on Development and Latin America, New York: Yale University Press.

ILO (2015) Advancing gender equality. The Co-operative Way. Geneva. 38p.

ILO. (nd.) Les cooperatives et le monde du travail ${ }^{\circ} 1$.

Johnson, S. (2015). Exploring conceptions of the social and solidarity economy: informal financial groups in Kenya. In Verschuur et al. (eds). Une économie solidaire peut-elle être féministe ? Homo oeconomicus, mulier solidaria. (p. 95-123). Paris : L'Harmattan.

Kabeer N. (1994) Reversed Realities: Gender Hierarchies in Development Thought. London: Verso.

Kabeer, N., (2010). Gender and Social Protection Strategies in the Informal Economy. New York et New Delhi : Routledge.

Kabeer, N., Sudarshan, R. \& Millward, K. (eds) (2013). Organising Women Workers in the Informal Economy. London: Zed Books.

Laclau, E. \& Mouffe, Ch. (2009). Hégémonie et stratégie socialiste: vers une politique démocratique radicale. Besançon : Les Solitaires intempestifs.

Lambert,A.(1993). "Les commerçantes maliennes du chemin de fer Dakar- Bamako", G. EMMANUEL, P. LABAZÉE (coord) Grands commerçants d'Afrique de l'Ouest. Logiques et pratiques d'un groupe d'hommes d'affaires contemporains, Paris, Karthala-ORSTOM, pp. 3770.

Laville, J.-L. (2013). Avec Mauss et Polanyi, vers une théorie de la démocratie et de l'économie plurielles, In I. Hillenkamp \& J.-L. Laville (éds.), Socioéconomie et démocratie. L'actualité de Karl Polanyi (pp. 271-300). Toulouse: Erès.

León,M. (1980). Mujer y capitalismo agrario. ACEP-Bogota.

Lipietz, A. (2002). Tiers secteur : un outil pour la libération des femmes, Mouvements, 19, 15.

Maruani, M. (1979). Les syndicats a l'ápreuve du féminisme. Syros.

Meillassoux C. (1984) La reproduction sociale. In Cahiers Internationaux de Sociologie, Vol. 77 (Juillet-Décembre 1984), pp. 383-395

Meillassoux, C. (1975). Femmes, greniers et capitaux. Paris, Maspéro.

Meillassoux. C. (1991). La leçon de Malthus: le contrôle démographique par la faim. In Les spectres de Malthus. Déséquilibres alimentaires, déséquilibres démographiques.

F.Gendreau, C. Meillassoux, B.Schlemmer, M. Verlet. Paris: ORSTOM-EDI-CEPED.

Mohanty, Ch. (1988). Under Western Eyes: Feminist Scholarship and Colonial Discourses. Feminist Review, 30, 61-88. 
Molyneux M. (2007) Organisations populaires et réseaux de solidarité de femmes : la redécouverte d'une ressource pour les politiques, in Verschuur Ch. (ed) Genre, mouvements populaires urbains et environnement. Cahiers genre et développement, $n^{\circ} 6$, Paris : L'Harmattan. pp. 385-403. 2007.

Narayan, L. \& Chikarmane, P. (2013). Power at the Bottom of the Heap: Organizing Waste Pickers in Pune. In Kabeer et al. (eds). Organising Women Workers in the Informal Economy, (p. XX). London: Zed Books

Ndoye, F. (2014). La restauration populaire, socle d'une solidarité pour la lutte contre l'insécurité alimentaire en milieu urbain Dakarois, Intervention au colloque "Homo oeconomicus, mulier solidaria". Une économie solidaire peut-elle être féministe ?, IHEID/IRD, Genève, 16-17 Octobre.

Nelson, J. (1993). The study of choice or the study of provisioning? Gender and the definition of economics. In Ferber, M. \& Nelson, J., (éd.). Beyond Economic Man : Feminist Theory and Economics. (p. 24-36). Chicago : University of Chicago Press.

Nobre, M. (2015). Economia solidaria, agroecologia y feminismo: prácticas para la autonomía en la organización del trabajo y de la vida. In C. Verschuur, I. Guérin, \& I. Hillenkamp (éds.), Une économie solidaire peut-elle être féministe ? Homo oeconomicus, mulher solidaria, pp. 273-294). Paris: L'Harmattan.

Nobre, M. \& Freitas, T. (2011). Possibilités et limites de la construction de l'égalité de genre dans l'économie solidaire. In I. Guérin, M. Hersent \& L. Fraisse (ed.), Femmes, économie et développement, Entre résistance et justice sociale, (p. 237-254). Paris : Erès/IRD.

Observatoire national de l'ESS-CNCRES, (2012). Atlas commenté de l'économie sociale et solidaire, Paris : Juris éditions - Dalloz.

Ostrom E. (2005). Understanding institutional diversity, Princeton University Press.

Polanyi, K. (1983). La Grande Transformation. Aux origines politiques et économiques de notre temps, Paris : Gallimard.

Postel-Coster, E. and Schrijvers, J. (eds) (1980). A Woman's Mind is Longer than a Kitchen Spoon. Report on Women in Sri Lanka. Research Project Women and Development, ColomboLeiden.

Prahalad, C. K., (2004). The Fortune at the Bottom of the Pyramid. Eradicating Poverty through Profits, Upper Saddle River NJ : Wharton School Publishing (WSP).

Prévost Héloïse, Galgani Silveira Leite Esmeraldo Gema, Guétat-Bernard Hélène, " II n'y aura pas d'agroécologie sans féminisme : I'expérience brésilienne. ", Pour 2/2014 (N²22), p. 275284

Rauber I. 2003. América Latina, Movimientos sociales y representación política. La HabanaMexico, Ed. Ciencias sociales, 117p.

Rauber, I. 2002, "Argentine: les femmes 'piqueteras'", Genre, mondialisation et pauvreté, Cahiers Genre et Développement n³, Paris-Genève, L'Harmattan, p.159-167

Rey, P.-P. e.a.,(1976), Capitalisme négrier, Paris, Maspéro.

Saiag, H. (2015). Monnaies locales et économie populaire en Argentine, Paris : Karthala. 
Saussey M. et Elias M., (2012. Consommer équitable et vendre son beurre. Imaginaire politicoécologique d'une filière globalisée. In, I. Guérin \& M. Selim (dir.), A quoi et comment dépenser son argent ?, (p. 207-228), Paris : L'Harmattan.

Saussey, M. (2013)._Développement. In C. Achin \& L. Bereni (dir.). Dictionnaire genre et science politique. Concepts, objets, problèmes. (p.154-167). Paris : Presses de la Fondation nationale des sciences politiques (FNSP).

Saussey, M. \& Degavre, F. (2015). Sécuriser l'existence des femmes et résister. Raisons d'agir des initiateurs et initiatrices d'organisations de l'économie sociale et solidaire, In Ch. Verschuur et al. (eds) Une économie solidaire peut-elle être féministe ? Homo oeconomicus, mulier solidaria. (p. 155-179). Paris : L'Harmattan.

Schrago S. (à paraître) Le fait religieux : sujets et objets dans un monde globalisé. In Journal des Anthropologues

Scott J.W. (1988). "Women's History", Past and Present, n¹01, p. 141-157, repris in Gender and the Politics of History, New York, Columbia University Press, traduit et publié en français dans Bisilliat, J. et Verschuur Ch . (dir.), 2000, «Genre, une catégorie utile d'analyse historique ", in Le Genre, un outil nécessaire, introduction à une problématique, Cahiers Genre et Développement, $n^{\circ} 1$, L'Harmattan, Genève - Paris, pp. 41-69.

Scott, J. W. (1986). "Gender: A useful category of historical analysis." The American Historical Review 91(5): 1053-1075.

Selim, M. (1998). Les politiques de l'islamisme au Bangladesh: entre libération et collaboration. In G. Heuzé \& M. Sélim, (dir.). Politique et religion dans I'Asie du Sud contemporaine. (p. 41-76). Paris : Karthala.

Sen, A. (2007). Shiv Sena Women. Violence and Communalism in Bombay Slum. Bloomington and Indianapolis: Indiana University Press.

Servet, J.-M. (2014). « De nouvelles formes de partage : la solidarité au delà de l'économie collaborative ". Institut Veblen pour les réformes économiques.

Solomon, C. (2013). Understanding the Dynamics of an NGO/MBO Partnership: Organizing and Working With Farm Women in South Africa, In N. Kabeer et al. (eds). Organising Women Workers in the Informal Economy. (p. XX). London: Zed Books.

de Sousa Santos, B. (2011). Épistémologies du Sud. Etudes rurales. 1(197), 21-49.

de Sousa Santos, B., Rodríguez Garavito, C. (2013). Alternatives économiques : les nouveaux chemins de la contestation. In I. Hillenkamp \& J.-L. Laville (éds.), Socioéconomie et démocratie. L'actualité de Karl Polanyi (pp. 127-147). Toulouse: Erès.

Sudarshan, R. (2015). Organising, gender, and solidarity: some reflections on Indian experiences. In Ch. Verschuur et al. (eds). Une économie solidaire peut-elle être féministe? Homo oeconomicus, mulier solidaria, (p. 123-138). Paris : L'Harmattan.

Tilly L. 1990. "Genre, histoire des femmes et histoire sociale". Tilly L. 1990. "Genre, histoire des femmes et histoire sociale". In Genèses. 148-166 In G Tilly L. 1990. "Genre, histoire des femmes et histoire sociale". In Genèses. 148-166enèses. 148-166

Tilly L., Scott J. (2012) Les femmes, le travail et la famille, Paris, Payot et Rivages, (1ère éd. 1978).

Tilly, C. (1978). From mobilisation to revolution, Addison/Wesley: Reading Press. 
Verschuur Ch. (2009). "Quel genre ? Résistances et mésententes autour du mot genre dans le développement", in Revue Tiers Monde n²00, oct-déc 2009, p.785-803, Paris, Armand Colin Verschuur, Ch. (2011). Mouvements et organisations populaires en milieu urbain : identités de genre et brèches pour le changement. In I. Guérin, M. Hersent \& L. Fraisse (ed.), Femmes, économie et développement, Entre résistance et justice sociale, (p. 185-206). Paris : Erès/IRD.

Verschuur, Ch., Guérin I. \& Hillenkamp I. (eds) (2015). Une économie solidaire peut-elle être féministe? Homo oeconomicus, mulier solidaria, Paris: L'Harmattan (Cahiers Genre et Développement).

Verschuur, Chr. (éd.). (2007). Genre, mouvements populaires urbains et environnement, Genève et Paris: Institut universitaire des études sur le développement (IUED), Espace femmes international (EFI) et L'Harmattan, coll. "Cahiers genre et développement ", $n^{\circ} 6$.

Verschuur, Chr. \& Destremeau B. (2012). Féminismes décoloniaux, genre et développement. Histoire et récits des mouvements de femme et des féminismes aux Suds. Revue Tiers Monde, 1(209), 7-18.

Waller W. et Jennings A. (1991), " A Feminist Institutionalist Reconsideration of Karl Polanyi ", Journal of Economic Issues, vol. 25, n², pp. 485-97.

Ypeij, A. (2002). Ateliers collectifs : une alternative pour les femmes dans les quartiers pauvres de Lima. In C. Verschuur and F. Reysoo (eds) Genre, mondialisation et pauvreté. Genève - Paris, L'Harmattan. 3: 99-105. 